\title{
PHYSICAL INTERPRETATION OF LIGHT CURVES OF R CORONAE BOREALIS-TYPE STARS
}

\author{
JACEK KRELOWSKI
}

Astronomical Institute of Nicolaus Copernicus University, Toruń, Poland

\begin{abstract}
The paper contains preliminary results of theoretical investigations of the light curves of RCB variables. A circumstellar dust envelope is assumed to be the source of the great visual opacity as well as the infrared and violet excesses. The processes of grain formation and the interaction with the radiation field are discussed briefly. The results of a laboratory experiment concerning the problem of the violet continuum are also described.
\end{abstract}

\section{Theoretical Model}

This contribution presents a very simple model of the $\mathbf{R}$ Coronae Borealis phenomenon.

Let us assume a spherically symmetric gaseous envelope thrown out from the star with a constant velocity. It follows from the depth of the minima that the mass of such a chemically unusual envelope may be of the order of $10^{26} \mathrm{gm}$. A layer of such a mass removed from a supergiant's surface has a mean optical depth of the order of $10^{-4}$ which can of course, be neglected. Let us denote by $r$ the outer radius of the envelope and its geometrical thickness by $\delta$ (Figure 1). The temperature distribution of an

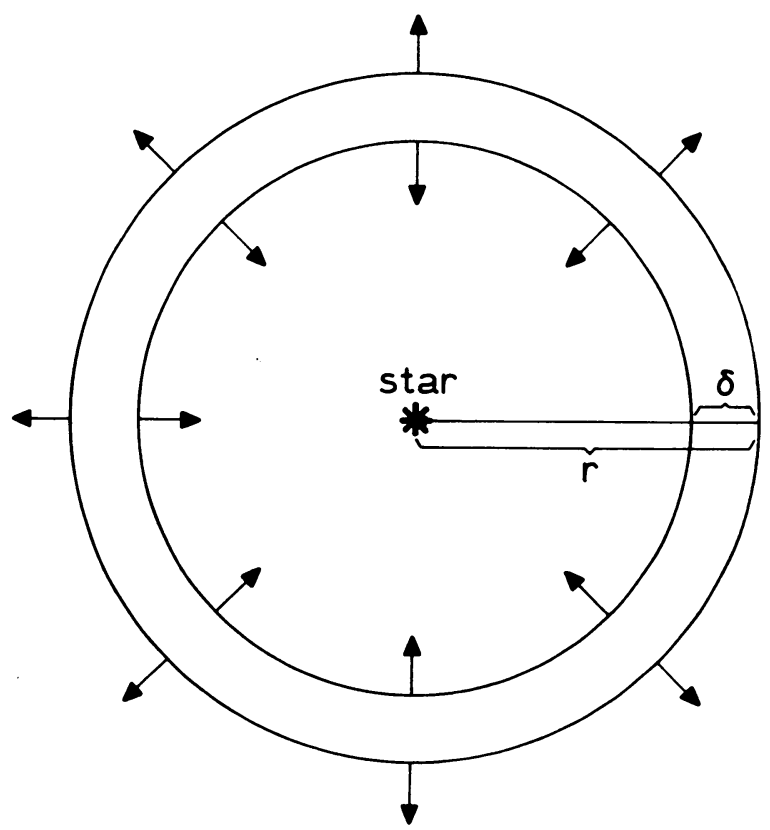

Fig. 1. The ejected envelope escaping from the central star. 
optically thin envelope is given by:

$$
T=0.8114 \mathrm{~T}_{\mathrm{e}}\left(R_{*} / r_{n}\right)^{1 / 2} .
$$

This is true, of course, before the dust is formed. $R_{*}$ is the radius of the central star and $r_{n}$ the radius of the considered point in the envelope.

Once the envelope is ejected, two forces appear tending to enlarge the thickness $\delta$. These forces originate from the following two accelerations: $a_{\mathrm{g}}$, the difference in gravitation at the edges of the envelope, and $a_{\mathrm{p}}$, connected with the action of the internal pressure.

and

$$
a_{\mathrm{g}}=g_{0} R^{2}\left[1 /(r-\delta)^{2}-1 / r^{2}\right]
$$

$$
a_{\mathrm{p}}=\frac{P_{1}-P_{\mathrm{N}}}{\bar{\varrho} \delta},
$$

where $g_{0}$ is the gravitation at the surface of the central star, $P_{1}$ and $P_{\mathrm{N}}$ are the pressures at the edges and $\bar{\varrho}$ is the mean mass density of the envelope. The expansion of the envelope is continuously decelerated by gravitation.

Let us divide the envelope into $N$ layers of equal masses. Their thicknesses are described by the following formulae:

$$
\begin{aligned}
\mathrm{d} x_{1} & =\left[(r-\delta)^{3}+\frac{3 M}{4 \pi m \varrho_{1}}\right]^{1 / 3}-(r-\delta), \\
\mathrm{d} x_{m} & =\left[\frac{3 M}{4 \pi m \varrho_{m}}-r^{3}\right]^{1 / 3}+r, \\
\mathrm{~d} x_{n} & =\left[r_{n}^{3}+\varrho_{n-1} \mathrm{~d} x_{n-1}\left(3 r_{n}^{2}+3 r_{n} \mathrm{~d} x_{n-1}+\mathrm{d} x_{n-1}^{2}\right) / \varrho_{n}\right]^{1 / 3}-r_{n},
\end{aligned}
$$

where $m$ is the number of pure gaseous layers, $n \leqslant m$ is the current subscript, and $M$ is the total mass of the gaseous envelope. Equations (4)-(6) are valid in the initial stage only; in other stages we can not obtain $\varrho_{1}$ and $\varrho_{m}$, only $\bar{\varrho}$ and we have to make another assumption; i.e.,

$$
\mathrm{d} x_{1}^{i}=\mathrm{d} x_{1}^{i-1} \delta^{i} / \delta^{i-1},
$$

where $i$ is the number of the evolution stage considered. The formulae (1)-(7) together with the perfect gas equation allow us to calculate the densities, temperatures and pressures.

Having all the physical parameters of the gas, we can obtain the partial pressures of the molecules assumed to be abundant in the considered envelope by the usual method. Basing our work on the calculations of Tsuji (1964) we consider only following molecules: $\mathrm{C}_{2}, \mathrm{C}_{3}, \mathrm{C}_{4}, \mathrm{C}_{5}, \mathrm{CO}, \mathrm{C}_{2} \mathrm{H}, \mathrm{C}_{3} \mathrm{H}, \mathrm{C}_{4} \mathrm{H}, \mathrm{CN}, \mathrm{C}_{2} \mathrm{~N}, \mathrm{C}_{3} \mathrm{~N}, \mathrm{HCN}, \mathrm{H}_{2}, \mathrm{~N}_{2}$. We are interested specially in the partial pressures of carbon molecules in order to examine the following problem: do any of them fulfill the necessary condition for the graphite formation in a gaseous cloud, i.e.

$$
P_{\mathrm{c}}>P_{\mathrm{v}},
$$


where $P_{\mathrm{c}}$ is the partial pressure of a carbon molecule and $\boldsymbol{P}_{\mathrm{v}}$ is the vapour pressure of this same molecule expressed by the simple temperature function,

$$
\log \left(P_{\mathrm{v}}\right)=B-A / T \quad A>0, B>0
$$

where $A$ and $B$ are the known constants compiled for carbon and published by Masser (1967). We did not try to identify the condensation sources of the graphite grains formed in the envelope as in many cases this is impossible even in a physical laboratory.

We can not obtain the shapes of grains from observations because neither the reddening of starlight nor the spectrum of the reradiated energy depend uniquely on grain shapes. In the laboratory, when no direction is specified (these conditions seem to be similar to those in the envelope), we always obtain spherical amorphic grains but, as it follows from the results of Lefèvre (1967) and Kamijo et al. (1973), small spheres tend to stick together forming chains. However we do not know anything about the process causing the above mentioned sticking; we believe that the forces joining grains into a chain are weaker than the intermolecular forces in a grain interior.

It seems possible that these chains can cause the polarization observed in $\mathrm{R} \mathbf{C r B}$ (Serkowski and Kruszewski, 1969; Coyne and Shawl, 1973) if they are aligned by the strongly anisotropic stellar radiation field according to the mechanism proposed by Harwit (1970). This hypothesis gives one a chance to interpret the dramatic changes in the radii of grains observed by Coyne and Shawl. Perhaps the chains are broken and the wavelength dependence of the polarization changes strongly for a given constant brightness before and after the minimum. As it follows from the above considerations, we can try to examine the model of the spherically symmetric envelope with spherical grains.

The growth rate of such a grain is represented by:

$$
\frac{\mathrm{d} a}{\mathrm{~d} t}=\Omega \sum i \alpha_{i}\left(P_{\mathrm{c}}^{i}-P_{\mathrm{v}}^{i}\right)\left(2 \pi m_{i} k T\right)^{-1 / 2},
$$

where $\Omega$ is the atomic volume in the solid state and $\alpha_{i}$ is the sticking coefficient for the $i$-atomic carbon molecule of mass $m_{i}$. (These coefficients are taken from measurements made by Thorn and Winslow, 1957.) Independently of (10) we obtain the mass fraction of the condensed carbon from the formula:

$$
\mathfrak{M} \%=\frac{1}{p(C)}\left[p(C)-p_{\mathrm{c}}-\frac{p_{\mathrm{c}} p_{\mathrm{o}}}{K_{\mathrm{co}}}-\frac{p_{\mathrm{c}} p_{\mathrm{n}}}{K_{\mathrm{cn}}}-\frac{p_{\mathrm{h}} p_{\mathrm{c}} p_{\mathrm{n}}}{K_{\mathrm{hen}} K_{\mathrm{cn}}}-\cdots\right],
$$

where the term in brackets contains all molecules with at least one atom of carbon. In (11) $p(C)$ is the fictitious pressure of carbon, $K_{x y}$ is a dissociation constant of an $x y$ molecule, and $p_{\mathrm{h}}, p_{\mathrm{o}}, p_{\mathrm{n}}$ are partial pressures of hydrogen, oxygen and nitrogen respectively; $p_{\mathrm{c}}$ is the saturated vapour pressure of carbon.

Using (11) we can calculate the total mass of grains and, because we have assumed their spherical shapes, also their total number. Substituting the real and imaginary parts of the graphite refraction index (measured by Taft and Phillipp, 1965) to the Mie formulae we obtain all efficiency factors of the grains under consideration. 
The light curves are calculated from the well known equation of transfer:

$$
\Delta m_{\lambda}=1.086 \pi a^{2} Q_{\text {ext }}(a, \lambda) N_{\mathrm{gr}},
$$

where $Q_{\text {ext }}$ is the efficiency factor for extinction and $N_{\mathrm{gr}}$ is the projected density of grains.

Let us now calculate the monochromatic fluxes of the envelope radiation. We have to know the grain temperature in each layer calculated from the following heat equation for a grain in the envelope:

$$
\begin{aligned}
& \left(\frac{R_{*}}{r_{n}}\right)^{2} \int_{0}^{\infty} \exp \left(-\tau_{\lambda}\right) B_{\lambda}\left(T_{\mathrm{e}}\right) Q_{\mathrm{abs}}(a, \lambda)\left[1+Q_{\text {sca }}(a, \lambda)\right] \mathrm{d} \lambda+ \\
& +\int_{0}^{\infty} \exp \left(-\tau_{\lambda}\right) B_{\lambda}\left(T_{\mathrm{g}}\right) Q_{\mathrm{abs}}(a, \lambda) \mathrm{d} \lambda=4 \int_{0}^{\infty} B_{\lambda}\left(T_{\mathrm{g}}\right) Q_{\mathrm{abs}}(a, \lambda) \mathrm{d} \lambda .
\end{aligned}
$$

The left hand side of this equation contains the terms due to absorption of the starlight (direct and scattered) attenuated by the extinction and diffused geometrically and absorption of the radiation of other grains, while the radiation of the grain under consideration is given on the right hand side. In (13) $\tau_{\lambda}$ is the optical depth, $B_{\lambda}$ is the Planck function, $Q_{\text {abs }}$ and $Q_{\text {sca }}$ are efficiency factors for absorption and scattering respectively and $T_{\mathrm{g}}$ is the grain temperature which is the only unknown parameter in this equation. In order to solve (13) we must know $r_{n}$ which may be obtained from the grain equation of motion:

$$
m_{\mathrm{gr}} \frac{\mathrm{d}^{2} r_{n}}{\mathrm{~d} t^{2}}=\left(R_{*} / r_{n}\right)^{2}\left(F_{\mathrm{rad}}-G\right)-F_{\mathrm{vis}}
$$

in which $m_{\mathrm{gr}}$ is the mass of the grain, $F_{\mathrm{rad}}$ and $F_{\mathrm{vis}}$ are radiation pressure and viscosity drag forces respectively, and

$$
G=m_{\mathrm{gr}} g_{0} .
$$

\section{Results of Computations}

The computations according to the above presented theory were performed using the Odra 1204 computer of Torun University and this section presents their results.

We have one completely free parameter which must be used in the computations the ejection velocity - as the first period of the envelope expansion is unobservable. Figure 2 presents three light curves constructed with the use of different ejection velocities; each of them is somewhat larger than the escape velocity of $55 \mathrm{~km} \mathrm{~s}^{-1}$. The curve computed using the smallest ejection velocity $\left(70 \mathrm{~km} \mathrm{~s}^{-1}\right)$ seems to be the best one. The brightness decreases in any case more rapidly than observed but it is impossible to use a smaller value for the ejection velocity. In such a case the accelerations $a_{\mathrm{p}}$ and $a_{\mathrm{g}}$ would act so long and make the gas pressure so low that the formation 


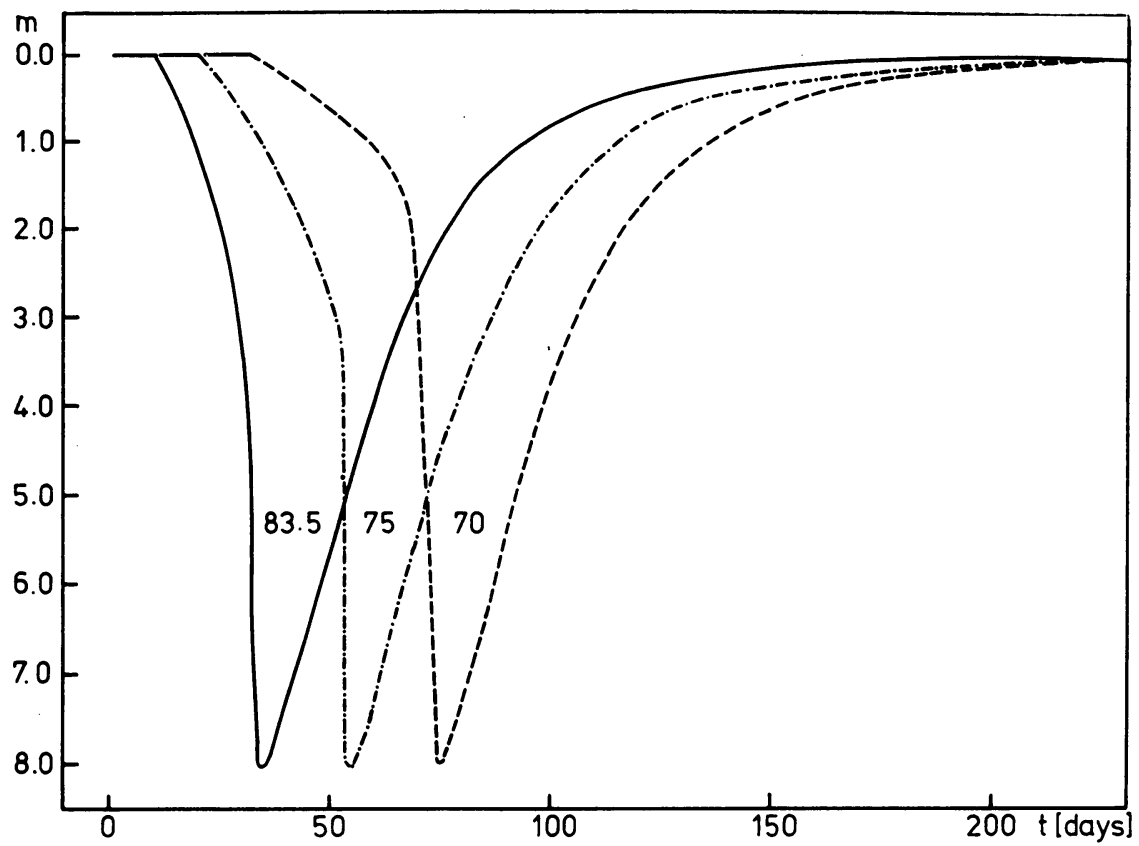

Fig. 2. Three light curves produced by the envelopes ejected with different velocities. (in $\mathrm{km} \mathrm{s}^{-1}$ ).

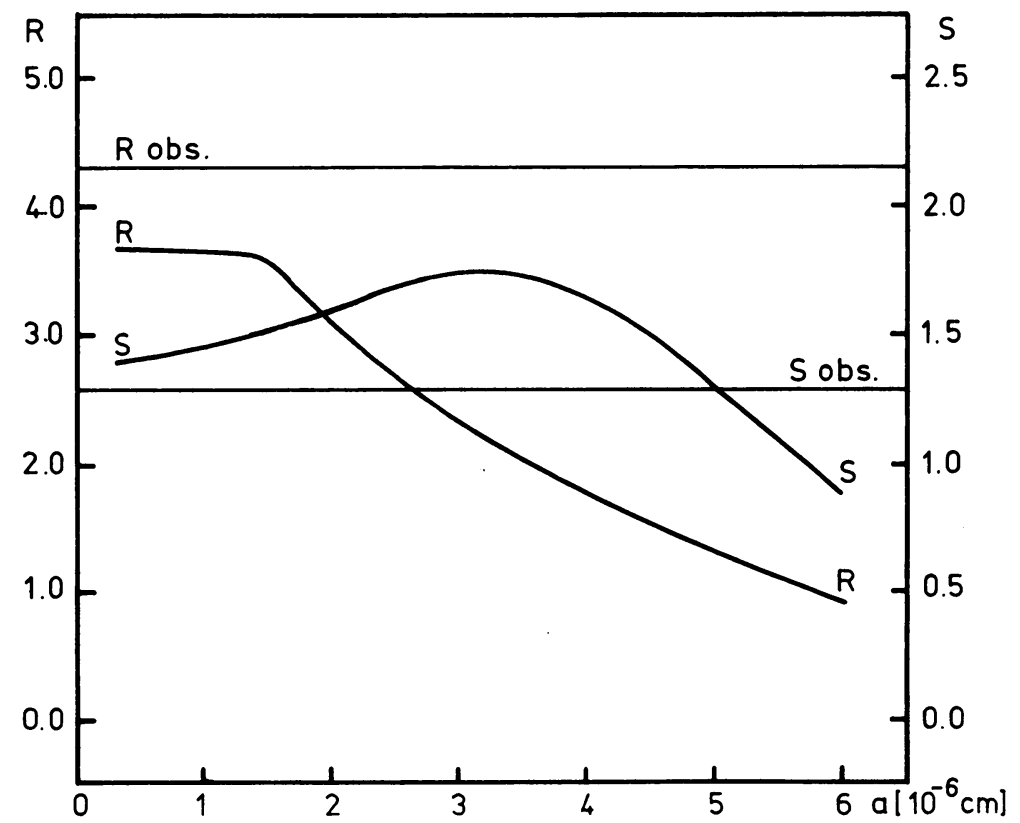

Fig. 3. Optical properties of graphite grains; $R=A_{V} / E_{B-V}$ and $S=E_{U_{-B}} / E_{B_{-} V}$ vs. grain radii. 


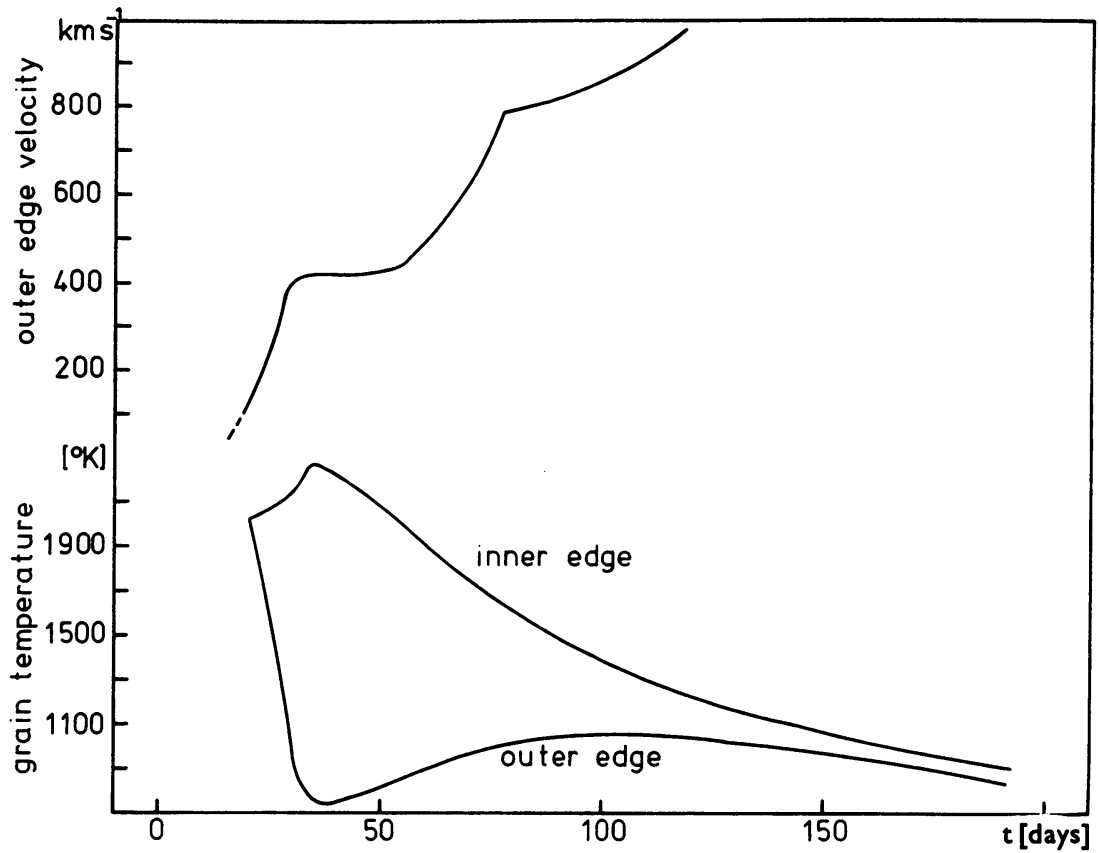

Fig. 4. The velocity of the outer envelope layer vs. time (above) and the temperatures of the outer and inner edges of the envelope (below). The largest temperature difference indicates the moment of minimum.

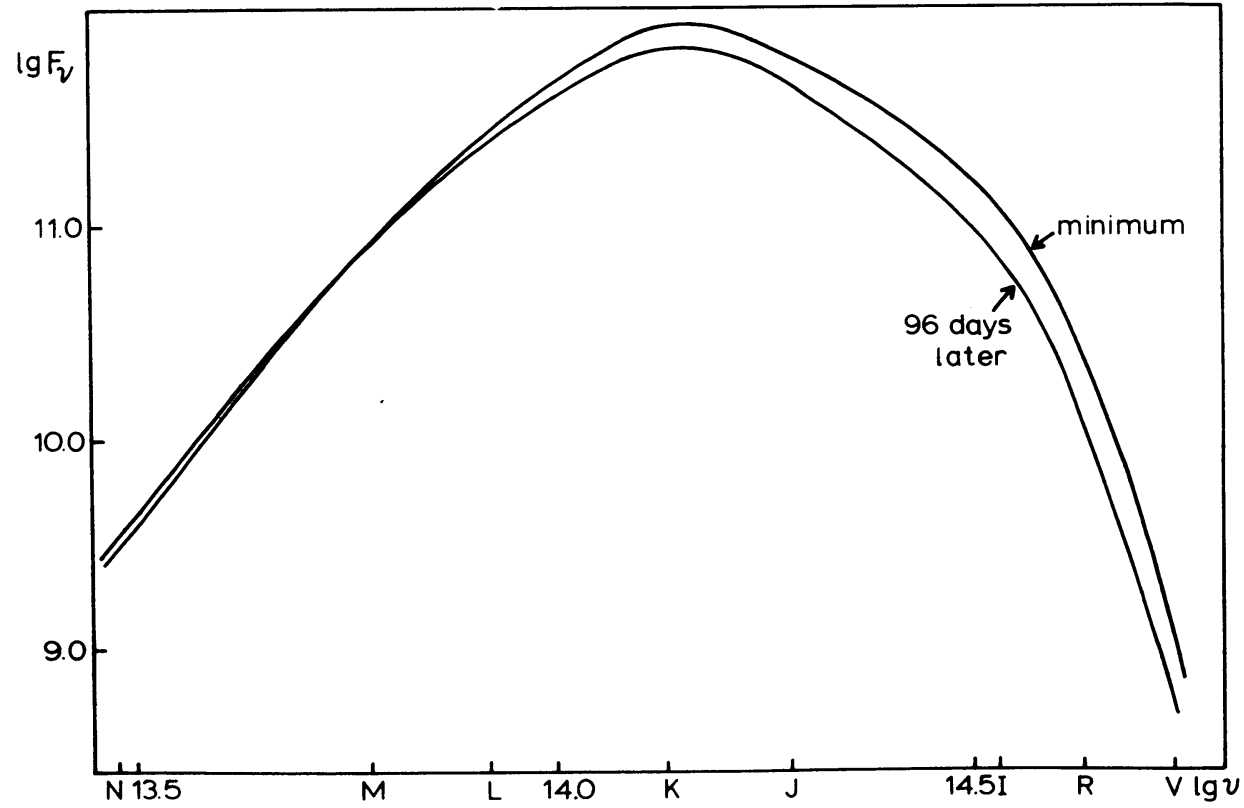

Fig. 5. The distribution of the energy produced by the envelope at the moment of minimum and 96 days later. 
of grains smaller than classical ones would be allowed (these grains can not explain the infrared excess). The radii of the grains which have been obtained by the calculations are about $4 \times 10^{-7} \mathrm{~cm}$ which is in accordance with the extinction law found by Alexander et al. (1972). It follows from Figure 3 that only very small grains can fit this law.

The variation of the velocity of the outer envelope layer together with the outer and inner envelope grain temperatures are drawn in Figure 4. At the moment of minimum there exists the largest difference between the extreme temperatures. In the late evolutionary stages the envelope becomes nearly isothermal. The result of the envelope cooling is the deviation of the energy distribution maximum to the red (Figure 5). Independently of it, the infrared flux levels for $\lambda \geqslant 3.6 \mu$ remain essentially unchanged which seems to be in accordance with observations (Lee, 1973). However we have not brought our calculations to very late stages of the envelope evolution. But the difference in the $V$ band between the minimum and the period 96 days later is as large as $7^{m} .5$.

The now-available observational data concerning the infrared behaviour of RCB stars are rather poor and the detailed quantitative comparison between the theory and observations seems to be impossible.

\section{Laboratory Investigations}

The above presented model does not describe the violet continuum observed in RY Sgr by Alexander et al. (1972). It is impossible to interpret this phenomenon neither by the radiation of a hot neighbour nor by the change of the physical parameters of the stellar atmosphere. Therefore we have to search for another source of this radiation in the envelope. The experiment made by Phillips and Brewer (1955) indicates the possibility of such an interpretation. They observed violet continuum for hot samples of graphite placed in vacuum. A similar experiment carried out in the Torun Department of Physics by Dr F. Rozpłoch and the author confirmed the results of Phillips and Brewer. It is drawn schematically in Figure 6. The heating

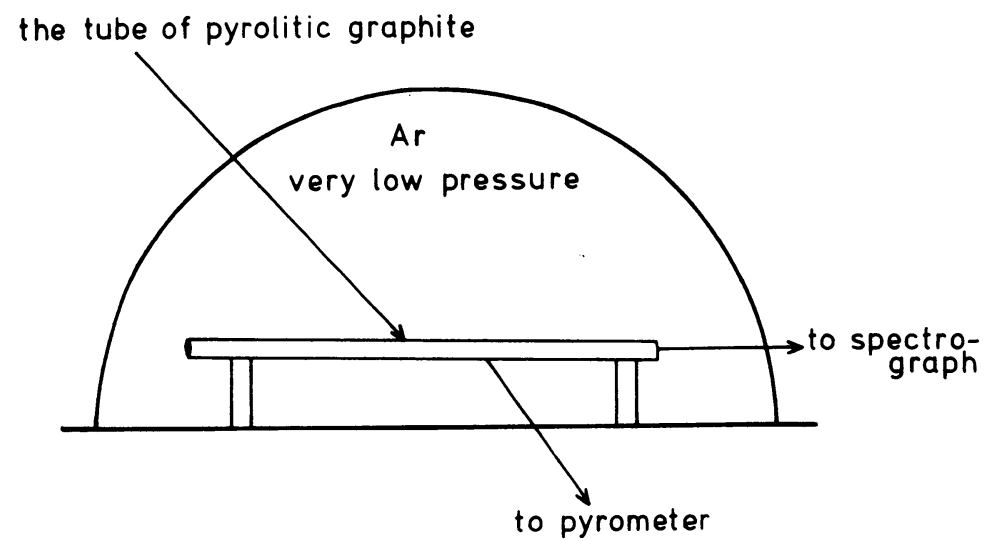

Fig. 6. The laboratory investigations of the violet continuum - a schematic diagram of the apparatus. 


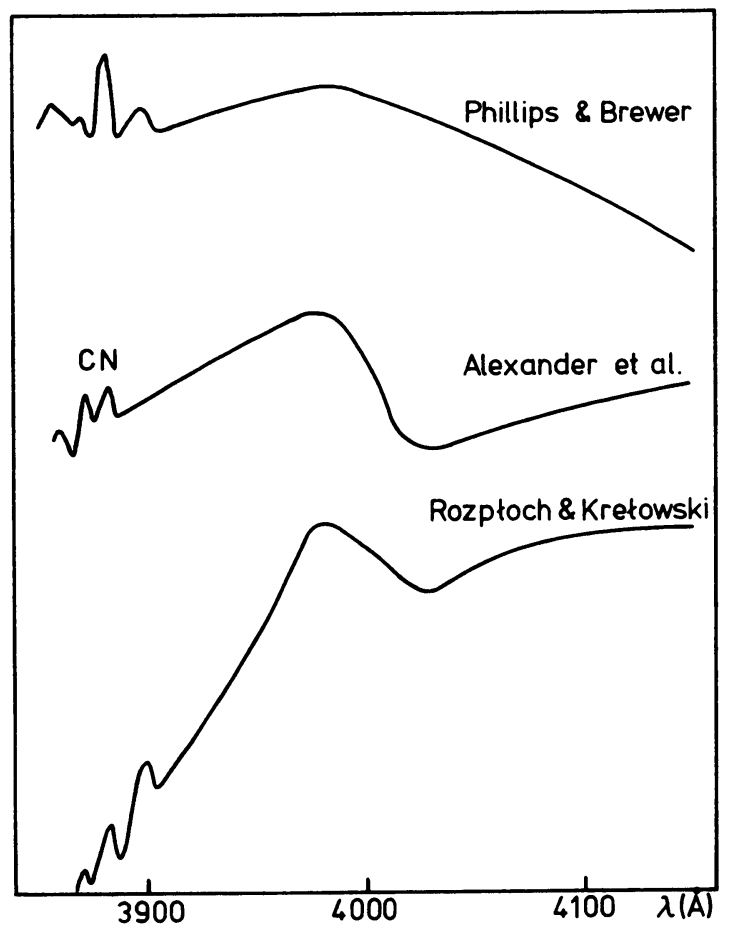

Fig. 7. The qualitative comparison of the observations of the violet continuum according to Alexander $e t$ al. with the experimental results mentioned in the text. The data were not published in absolute units, so there is no scale on the $y$-axis.

resistance tube was placed in a very low pressure argon atmosphere. Its temperature was measured with the aid of a pyrometer. The spectrograph was directed along the long axis of the tube, not at its hot walls. (We have to mention that the sensitivity of the photomultiplier in our spectrograph falls rapidly at short wavelengths and reaches zero at about $3800 \AA$ ). The continuum appeared when the temperature reached as large a value as about $2800 \mathrm{~K}$. The violet continuum was not produced by the tube itself but rather by the graphite sublimation products in the neighbourhood of solid graphite. The comparison of our results with those of Phillips and Brewer and observations of Alexander $e t$ al. are given in Figure 7. It is of course only a qualitative comparison as the physics of this process is very ambiguous up to now.

\section{References}

Alexander, J. B., Andrews, P. J., Catchpole, R. M., Feast, M. W., Lloyd Evans, T., Menzies, J. W., Wisse, P. N. J., and Wisse, M.: 1972, Monthly Notices Roy. Astron. Soc. 158, 305.

Coyne, G. V. S. and Shawl, S. J.: 1973, Astrophys. J. 186, 961.

Harwit, M.: 1970, Nature 226, 61.

Kamijo, F., Nakada, Y., Iguchi, T., Fujimoto, M., Takada, M., and Takajo, S.: 1973, in J. M. Greenberg and H. C. van de Hulst (eds.), 'Interstellar Dust and Related Topics', IAU Symp. 52, 323. 
Lefèvre, J.: 1967, Ann. Astrophys. 30, 731.

Lee, T. A.: 1973, Publ. Astron. Soc. Pacific 85, 637.

Masser, C.: 1967, NASA TN D-4147.

Phillips, J. G. and Brewer, L.: 1955, Mem. Soc. Roy. Liège 15, 341.

Serkowski, K. and Kruszewski, A.: 1969, Astrophys. J. 155, L15.

Taft, E. A. and Phillipp, H. R.: 1965, Phys. Rev. 138A, 197.

Thorn, R. and Winslow, G.: 1957, J. Chem. Phys. 26, 186.

Tsuji, T.: 1964, Ann. Tokyo Astron. Obs. 9, No. 1.

\section{DISCUSSION}

A. Z. Dolginov: What mechanism for the orientation of the grains did you use? It seems to me that Harwit's mechanism needs too long a time for the orientation.

$J$. Krelowski: In the present preliminary context I have not considered the polarization. Only the possibility of such a mechanism in RCB -star envelopes was mentioned. 